\title{
Trophic Effects of Mesenchymal Stem Cells in Chondrocyte Co-Cultures are Independent of Culture Conditions and Cell Sources
}

\author{
Ling Wu, M.Sc., ${ }^{1}$ Henk-Jan Prins, Ph.D., ${ }^{2,3}$ Marco N. Helder, Ph.D., ${ }^{4}$ \\ Clemens A. van Blitterswijk, Ph.D., ${ }^{1}$ and Marcel Karperien, Ph.D., ${ }^{1, *}$
}

Earlier, we have shown that the increased cartilage production in pellet co-cultures of chondrocytes and bone marrow-derived mesenchymal stem cells (BM-MSCs) is due to a trophic role of the MSC in stimulating chondrocyte proliferation and matrix production rather than MSCs actively undergoing chondrogenic differentiation. These studies were performed in a culture medium that was not compatible with the chondrogenic differentiation of MSCs. In this study, we tested whether the trophic role of the MSCs is dependent on culturing co-culture pellets in a medium that is compatible with the chondrogenic differentiation of MSCs. In addition, we investigated whether the trophic role of the MSCs is dependent on their origins or is a more general characteristic of MSCs. Human BM-MSCs and bovine primary chondrocytes were co-cultured in a medium that was compatible with the chondrogenic differentiation of MSCs. Enhanced matrix production was confirmed by glycosaminoglycans (GAG) quantification. A species-specific quantitative polymerase chain reaction demonstrated that the cartilage matrix was mainly of bovine origin, indicative of a lack of the chondrogenic differentiation of MSCs. In addition, pellet co-cultures were overgrown by bovine cells over time. To test the influence of origin on MSCs' trophic effects, the MSCs isolated from adipose tissue and the synovial membrane were co-cultured with human primary chondrocytes, and their activity was compared with BM-MSCs, which served as control. GAG quantification again confirmed increased cartilage matrix production, irrespective of the source of the MSCs. EdU staining combined with cell tracking revealed an increased proliferation of chondrocytes in each condition. Irrespective of the MSC source, a short tandem repeat analysis of genomic DNA showed a decrease in MSCs in the co-culture over time. Our results clearly demonstrate that in co-culture pellets, the MSCs stimulate cartilage formation due to a trophic effect on the chondrocytes rather than differentiating into chondrocytes, irrespective of culture condition or origin. This implies that the trophic effect of MSCs in co-cultures is a general phenomenon with potential implications for use in cartilage repair strategies.

\section{Introduction}

D ESPITE THE SUCCESS of autologous chondrocyte implantation in treating large-size cartilage defects, there are some disadvantages of this treatment that limit its broader clinical application. One major issue is the requirement of relatively large quantities of chondrocytes from the patient, ${ }^{1}$ which are obtained by in vitro expansion. The partial replacement of the chondrocytes with mesenchymal stem cells (MSC) has been proposed to tackle this problem. Many studies have evaluated the feasibility of this idea by the co- culturing of MSCs and chondrocytes. ${ }^{2}$ Indeed, cartilage matrix deposition was found to be improved in the cocultures of MSCs and chondrocytes compared with the cultures of pure chondrocytes or MSCs. ${ }^{3,4}$ In our previous report, ${ }^{5}$ we have shown that pellet co-cultures of bovine primary chondrocytes (bPCs) and human MSCs benefit cartilage matrix formation and that cartilage matrix genes were mainly expressed by bovine chondrocytes. In addition, we also showed that the ratio of MSCs decreased dramatically due to the massive cell death of MSCs by apoptosis. Chondrocyte proliferation was increased by either co-culturing

\footnotetext{
${ }^{1}$ Department of Tissue Regeneration, MIRA-Institute for Biomedical Technology and Technical Medicine, University of Twente, Enschede, The Netherlands.

${ }^{2}$ Department of Oral Cell Biology, Academic Centre for Dentistry Amsterdam, Amsterdam, The Netherlands.

Departments of ${ }^{3}$ Oral and Maxillofacial Surgery and ${ }^{4}$ Orthopaedics, VU Medical Center, Amsterdam, The Netherlands.

*Current affiliation: Department of Developmental BioEngineering, MIRA-Institute for Biomedical Technology and Technical Medicine, University of Twente, Enschede, The Netherlands.
} 
with MSCs or culturing with MSCs conditioned medium. These findings were also confirmed by an independent study performed by Acharya et al.(2011). ${ }^{6}$ This and our study demonstrated a new mechanism of cellular interaction in the co-culture of MSCs and chondrocytes, in which the trophic effects of MSCs stimulate chondrocyte proliferation and cartilage matrix deposition rather than actively undergoing chondrogenic differentiation.

The concept of MSCs as a trophic mediator of tissue repair is introduced by Arnold Caplan (2006), ${ }^{7}$ who borrowed the term trophic from neurobiology, in which it refers to the bioactive molecules produced by nerve terminals, which are not neurotransmitters. ${ }^{8}$ In relation to MSCs, the term trophic was first used to describe the process in which MSCs secrete factors that stimulate nearby cells to release functionally bioactive molecules. ${ }^{7}$ Later, the term also related to the effect of the factors produced by MSC on the viability, proliferation, and matrix production of the neighboring cells. This concept has resulted in a paradigm shift in the way MSCs are involved in tissue repair. While traditionally it was believed that MSCs mainly repair damaged tissue by differentiating into specific cell types and replacing lost cells, ${ }^{9}$ nowadays, the trophic role of the MSC in tissue repair is considered more important. ${ }^{10}$ It was reported that MSCs, when introduced either directly or systemically into stroked brain of rats, promote the gain of coordinated functions without differentiating into neurons or any other supporting cell type. ${ }^{11}$ Other examples are from the trophic role of MSCs in stimulating cardiomyocyte proliferation in vitro, ${ }^{12}$ and vascular regeneration in vivo. ${ }^{13}$

So far, most of the reports dealing with the trophic effects of MSCs are based on studies using bone marrow-derived MSCs (BM-MSCs). There are many more sources from which MSCs can be isolated. For examples, adipose tissue can give rise to multipotent stromal cells, ${ }^{14,15}$ and the human synovial membrane also contains an MSC population. ${ }^{16}$ So far, MSCs have been isolated from many other tissues. ${ }^{17,18}$ The MSCs isolated from other sources share many common features with BM-MSCs, ${ }^{19,20}$ and have been applied in tissueengineering strategies. ${ }^{21-25}$ Nevertheless, only a few papers documented the paracrine or autocrine effects of adipose tissue-derived MSCs in tissue regeneration. ${ }^{26,27}$ The trophic effects of MSCs isolated from a nonbone marrow origin have not yet been systematically and thoroughly studied.

In a previous study, we had co-cultured human BM-MSCs and bPCs in pellets in a chondrocyte proliferation medium, ${ }^{5}$ and showed that increased cartilage matrix formation was not due to the chondrogenic differentiation of MSCs. These data indicated that in pellet co-cultures, signals from the chondrocytes alone are insufficient to induce the chondrogenic differentiation of MSCs, at least under these culture conditions. In this study, we performed experiments in which BM-MSCs and bPCs were co-cultured in a chondrogenic differentiation medium to investigate the influence of growth factors in the culture medium on MSCs' trophic effects. In addition, we examined whether MSCs from multiple sources (bone marrow, adipose tissue, and synovial membrane) have similar trophic effects by co-culturing them with human primary chondrocytes (hPCs). Our data presented here demonstrated that the trophic effects of MSCs on chondrocytes in pellet co-cultures is a general feature of MSCs, independent of culture conditions and their source.

\section{Materials and Methods}

\section{Cell culture and expansion}

bPCs were isolated from full-thickness cartilage of knee biopsies from cows that were approximately 6 months old. hPCs were obtained from macroscopically healthy cartilage obtained from the knee or hip biopsies of patients with endstage osteoarthritis undergoing total knee or hip replacement. Both bovine and human cartilages were digested for $20-22 \mathrm{~h}$ in collagenase type II $(0.15 \%)$ dissolved in chondrocytes proliferation medium. The components of the chondrocytes proliferation medium are Dulbecco's Modified Eagle Medium (DMEM) supplemented with 10\% fetal bovine serum, $1 \times$ nonessential amino acids, $0.2 \mathrm{mM}$ ascorbic acid 2-phosphate (AsAP), $0.4 \mathrm{mM}$ proline, $100 \mathrm{U} / \mathrm{mL}$ penicillin, and $100 \mu \mathrm{g} / \mathrm{mL}$ streptomycin. More details about chondrocyte isolation are described elsewhere. ${ }^{28}$ Bone marrow mesenchymal stem cells (BM-MSCs) were separated from human bone marrow aspirates as previously described. ${ }^{29}$ Human adipose tissue mesenchymal stem cells (AT-MSCs) and synovium MSCs were isolated according to the procedures in previous publications. ${ }^{30-31}$ MSCs from all kinds of sources were cultured in MSC proliferation medium ( $\alpha$-MEM supplemented with $10 \%$ fetal bovine serum, $1 \%$ L-glutamin, $0.2 \mathrm{mM}$ ascorbic acid, $100 \mathrm{U} / \mathrm{mL}$ penicillin, $10 \mu \mathrm{g} / \mathrm{mL}$ streptomycin, and $1 \mathrm{ng} / \mathrm{mL}$ basic fibroblast growth factor).

The use of all human materials in this study has been approved by a local Medical Ethics Committee. All reagents used for cell culture were purchased from Gibco, Invitrogen, unless otherwise stated. Common chemicals were purchased from Sigma-Aldrich.

\section{Pellet culture and chondrogenic differentiation}

For mono-cultures, 200,000 cells of hPCs or MSCs were seeded in one well of a round-bottom 96-well plate (nontissue culture treated). For the co-cultures, 200,000 cells were seeded in an $80 \% \mathrm{MSC} / 20 \%$ hPC or bPC ratio. The cells were initially seeded in a chondrocyte proliferation medium and centrifuged for $5 \mathrm{~min}$ at $500 \mathrm{~g}$. The medium was changed to a chondrogenic differentiation medium (DMEM supplemented with $40 \mu \mathrm{g} / \mathrm{mL}$ of proline, $50 \mathrm{ug} / \mathrm{mL}$ of ITS-premix, $50 \mathrm{ug} / \mathrm{mL}$ of AsAP, $100 \mathrm{ug} / \mathrm{mL}$ of Sodium Pyruvate, $10 \mathrm{ng} /$ $\mathrm{mL}$ of TGF $\beta 3,10^{-7} \mathrm{M}$ of dexamethasone, $500 \mathrm{ng} / \mathrm{mL}$ of BMP6, 100U of penicillin/mL, and $100 \mu \mathrm{g} / \mathrm{mL}$ of streptomycin) 1 day after seeding when the stable pellets had been formed. The cell pellets were cultured for 4 weeks before the analysis.

\section{Histology}

The cell pellets were fixed with $10 \%$ formalin for $15 \mathrm{~min}$, dehydrated with ethanol, and embedded in paraffin using routine procedures. A microtome was used to cut $5 \mu \mathrm{m}$-thick sections. The slides were then deparaffinized and stained for sulfated glycosaminoglycans (GAG) with Alcian blue. Nuclei were counterstained with nuclear fast red.

\section{Quantitative GAG and DNA assay}

Cell pellets $(n=6)$ were washed with phosphate-buffered saline (PBS) and stored at $-80^{\circ} \mathrm{C}$ for $16-20 \mathrm{~h}$. Subsequently, they were digested in digestion buffer $(1 \mathrm{mg} / \mathrm{mL}$ proteinase 
$\mathrm{K}$ in Tris/ethylenediaminetetraacetic acid (EDTA) buffer (pH 7.6) containing $18.5 \mu \mathrm{g} / \mathrm{mL}$ iodoacetamide and $1 \mu \mathrm{g} / \mathrm{mL}$ pepstatin A) for more than $16 \mathrm{~h}$ at $56^{\circ} \mathrm{C}$. The GAG content was spectrophotometrically determined with 1,9dimethylmethylene blue chloride (DMMB) staining in PBE buffer $\left(14.2 \mathrm{~g} / \mathrm{L} \mathrm{Na}_{2} \mathrm{HPO}_{4}\right.$ and 3.72g/L Na${ }_{2}$ EDTA, pH 6.5) using an ELISA reader (TECAN) at an absorbance of $520 \mathrm{~nm}$ with the chondroitin sulfate as a standard. Cell numbers were determined by the quantification of total DNA using a CyQuant DNA Kit (Molecular Probes).

\section{DNA isolation, RNA isolation, and quantitative polymerase chain reaction}

DNA samples of pellets were extracted with the DNA Mini Kit (Promega) according to the manufacturer's protocols. RNA samples of cell pellets were isolated with the NucleoSpin RNA II Kit (Macherey-Nagel). Total RNA was reverse transcribed into cDNA using the iScript cDNA Synthesis kit (Bio-Rad). Quantitative polymerase chain reaction (qPCR) was performed on genomic DNA or cDNA samples by using the iQ SYBR Green Supermix (Bio-Rad). PCR reactions were carried out on the MyiQ2 Two-Color Real-Time PCR Detection System (Bio-Rad) under the following conditions: cDNA was denatured for $5 \mathrm{~min}$ at $95^{\circ} \mathrm{C}$, followed by 45 cycles, consisting of $15 \mathrm{~s}$ at $95^{\circ} \mathrm{C}, 15 \mathrm{~s}$ at $60^{\circ} \mathrm{C}$, and $30 \mathrm{~s}$ at $72^{\circ} \mathrm{C}$. For each reaction, a melting curve was generated to test primer dimer formation and nonspecific priming. The sequences of the primers for real-time PCR, either species specific or cross-species specific, were as previously described. ${ }^{5}$ For the PCR on genomic DNA, standard curves were generated by using the serial dilutions of genomic DNA as a template. Bio-Rad iQ5 optical system software (version 2.0) was used to calculate copy numbers for each condition using the standard curves as reference. The relative signal was defined as the proportion of human or bovine GAPDH copy numbers as the percentage of the total copy numbers of both human and bovine genes. The calculation of relative expression was performed with the Bio-Rad iQ5 optical system software (version 2.0) using the double delta Ct method. ${ }^{32}$ Cross-species-specific GAPDH primers were used for normalization.

\section{Cell tracking with organic fluorescent dyes}

The organic fluorescent dye CM-DiI (Molecular Probes) was used for cell tracking in co-cultures. The cells were labeled according to the manufacturer's protocol. Briefly, the cells were trypsinized and resuspended in PBS at a concentration of $2 \times 10^{6}$ cells $/ \mathrm{mL}$. The cells were incubated with $4 \mu \mathrm{M}$ of $\mathrm{CM}$-DiI at $37^{\circ} \mathrm{C}$ for $5 \mathrm{~min}$, followed by an incubation at $4^{\circ} \mathrm{C}$ for $15 \mathrm{~min}$. The cells were washed with PBS and applied in co-culture experiments.

\section{5-ethynyl-2'-deoxyuridine labeling and staining}

Cell proliferation in pellets was examined with the Click$\mathrm{iT}^{\circledR}$ EdU Imaging Kit (Molecular Probes). The cell pellets were cultured in a chondrogenic differentiation medium containing $10 \mu \mathrm{M}$ EdU (5-ethynyl-2'-deoxyuridine) for $24 \mathrm{~h}$ before harvesting. At day 2, the cell pellets were washed with PBS and fixed with $10 \%$ formalin for $15 \mathrm{~min}$. Sections of $10 \mu \mathrm{m}$ were cut with a cryotome. The sections were per- meabilized with $0.5 \%$ of Triton $\times 100$ for 20 min and stained for EdU with Alexa 488 cocktail. Nuclei were counterstained with Hoechst 33342 (provided in the same kit).

\section{Image acquisition and analysis}

Histological images were acquired with a Nikon E300 microscope. Fluorescent images were taken with a BD pathway 435 confocal microscope (BD Biosciences). Details of image quantification were published elsewhere. ${ }^{5}$ Values represent the mean \pm standard deviation of at least 3 biological replicates.

\section{Short tandem repeats analysis}

Genomic DNA samples were extracted from pellets with the QIAamp DNA Mini Kit (Qiagen). The sixteen loci of the kit PowerPlex 16 System (Promega) were amplified, typed, sequenced, and analyzed by ServiceXS B.V.. Specific alleles for the donor of hMSCs and the donor of hPCs were found in several loci. These alleles were used to define the origin of cells in the allogeneic co-culture of hMSCs and hPCs. The amount of DNA present for each donor was calculated from the areas of the electropherogram for each locus of the hMSCs' or hPCs' specific alleles, and the ratio of hMSCs and hPCs was determined.

\section{Statistical analysis}

GAG and DNA quantifications were examined for statistical significance with the one-way analysis of variance followed by the Tukey's Honestly Significant Difference Test. Statistical analysis of EdU-positive cells was performed by using the Student's $t$ test. $p$-Values of $<0.05$ were considered statistically significant.

\section{Results}

\section{Co-culture pellets in chondrogenic differentiation medium show increased cartilage formation}

To test whether growth conditions, favorable for the chondrogenic differentiation of MSCs, affect the trophic effects of MSCs in pellet co-cultures, we examined the cartilage matrix formation of BM-MSCs and bPCs in a chondrogenic differentiation medium. Each of the 3 BM-MSC donors were fully capable of differentiating into the chondrogenic, osteogenic, and adipogenic lineage and expressed typical cell surface markers of MSCs (data not shown), although some variability was observed in the chondrogenic differentiation potential. After 4 weeks of co-culture, histology and GAG assays were performed to evaluate cartilage formation. As shown in Figure 1A, the presence of GAG is indicated by Alcian blue staining in both mono-cultures and co-culture pellets. Cells in the positively stained areas were embedded in lacunae and showed typical characteristics of chondrocytes, round shape, and the presence of an Alcian bluepositive extracellular matrix.. Similar data were obtained using Toluidine blue staining (data not shown). Quantitative analysis showed that both total GAG and total DNA increased as the ratio of MSCs decreased (Fig. 1B). In line with previous studies, the co-culture pellets contained more GAG than the pellets seeded with $100 \%$ chondrocytes, when normalized to the initial seeding percentage of bPCs, indicating 


\section{A $\quad$ BM-MSC/bPC}

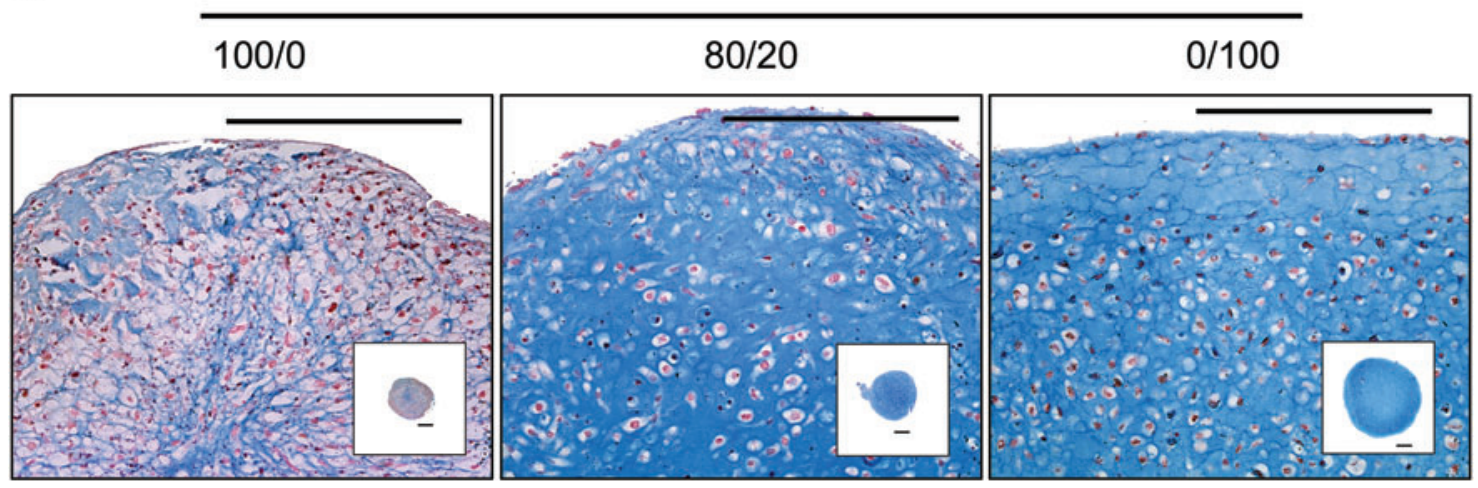

B

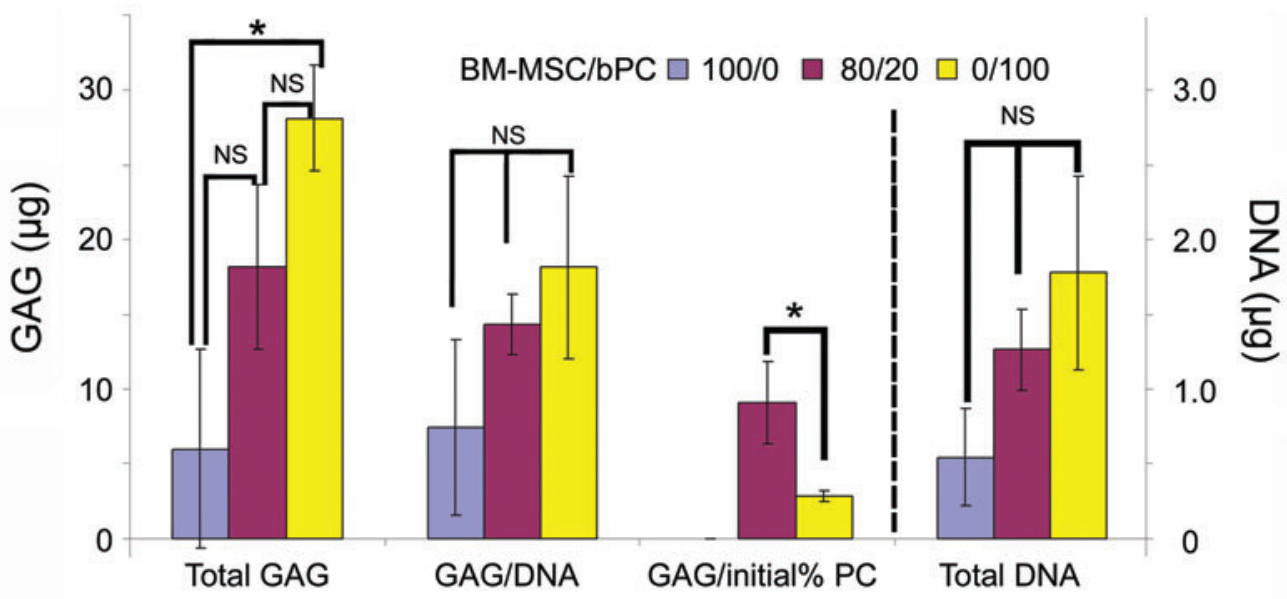

FIG. 1. Cartilage matrix formation is increased in co-culture pellets cultured in a chondrogenic differentiation medium. Pellets (200.000cells) of BM-MSCs from 3 donors and bPC in 3 different ratios (100:0; 80:20 and 0:100 of BM-MSC:bPC) were cultured in a medium that was compatible with the chondrogenic differentiation of MSCs. After 4 weeks, the pellets were collected for a histological analysis. (A) Alcian blue staining was performed to examine the deposition of sulfated GAGs in midsagittal paraffin sections. Inserts indicate the overview of each pellet. Pictures show the results of a representative donor of MSCs, while 3 donors were tested. Scale bar $=100 \mu \mathrm{m}$. (B) GAG and DNA of cell pellets $(n=6$ per donor) were quantitatively measured 4 weeks after the co-culture. Averages of 3 donors of BM-MSCs are shown. Ratios of hMSCs and bPCs are indicated by the bar colors. The left $y$-axis is for "Total GAG," "GAG/DNA," and "GAG/initial\%PC," while the right $y$-scale is for "Total DNA." Asterisk represents $p<0.05$. NS, Not Significant. Data are presented as a mean \pm standard deviation (SD). BM-MSCs, bone marrow-derived mesenchymal stem cells; bPC, bovine primary chondrocyte; GAG, glycosaminoglycans. Color images available online at www.liebertonline.com/tea

that the beneficial effect on cartilage matrix formation in cocultures is preserved when the pellets are cultured in growth factors-containing medium.

\section{Proliferation of chondrocytes causes ratio changes of two cell types in pellets cultured in chondrogenic differentiation medium}

Species-specific qPCR was performed to study the origin of the chondrogenic marker genes in co-culture pellets (Fig. 2A). At week 4, the expression levels of both human Aggrecan $(A C A N)$ and human Collagen 2 (COL2) were much lower in co-culture pellets than in pure MSCs pellets. On the other hand, the expression levels of bovine ACAN or COL2 in co-culture pellets were either higher or similar to those of pure bPCs pellets. These data indicated that the cartilaginous matrix in co-culture pellets is mainly of bovine origin. At week 4 of culture, genomic DNA was isolated from the cell pellets, and used as a template in species-specific qPCR. The ratio of human/bovine cells dropped from $80 \%$ (initial seeding percentage after 1 day) to approximately $40 \%$ after 4 weeks of culture (Fig. 2B).

Cell proliferation in co-culture pellets was studied using EdU incorporation combined with cell tracking. For this, bovine chondrocytes were labeled with CM-Dil (red). Proliferation was studied at day 2. As shown in Figure 2C, the EdU-positive cells were mainly found at the periphery of the pellets. Cell tracking showed that co-cultures predominantly increased the proliferation of the chondrocytes with a minor effect on the proliferation of the MSCs (Fig. 2D).

\section{Co-culture of hPCs and MSCs from multiple sources increases cartilage matrix formation}

To investigate whether the source of MSCs influences the degree of cartilage formation in co-culture pellets, MSCs derived from human adipose tissue and synovium were used in co-culture pellets with hPCs, while BM-MSCs served as a control. Alcian blue staining shows the deposition of GAGs, to some extent, in all groups (Fig. 3). GAG and DNA 
A

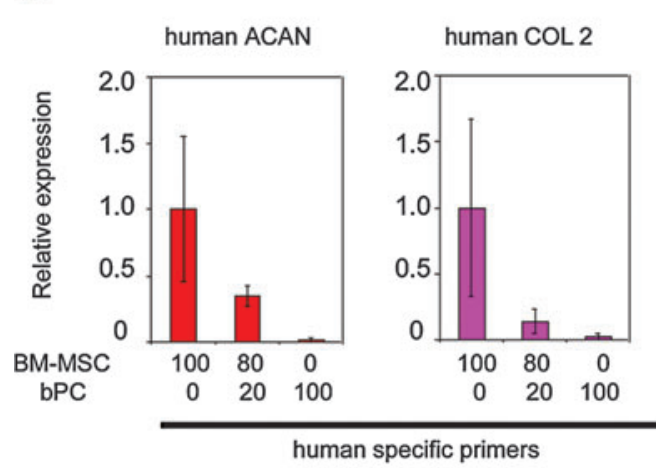

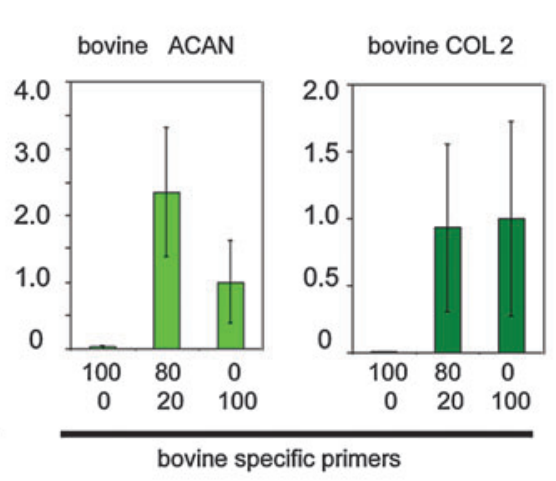

B

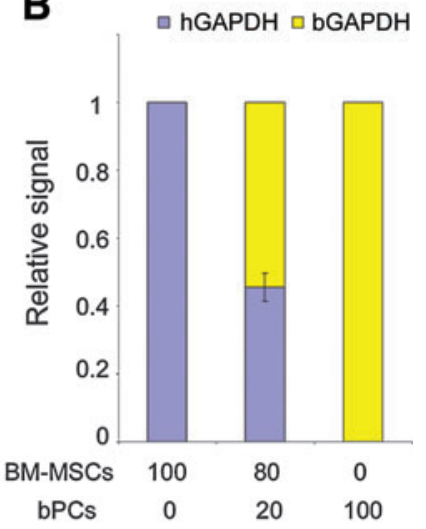

C

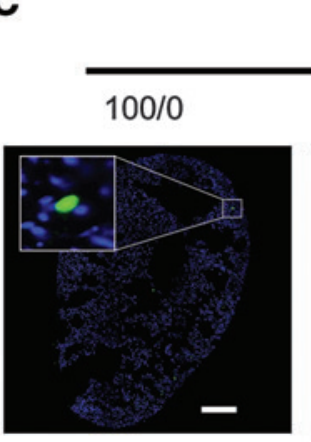

BM-MSCs/bPCs

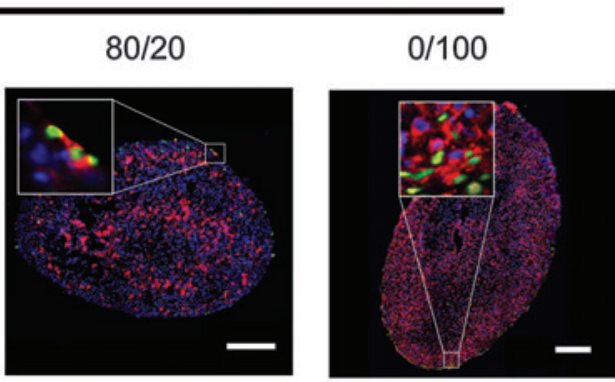

D

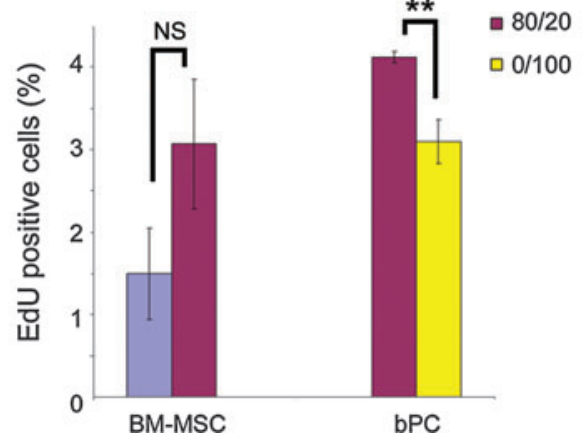

FIG. 2. Enhanced cartilage matrix formation is caused by trophic effects of MSCs. Co-culture pellets of BM-MSCs and bPC were established and cultured in a chondrogenic medium as previously described. (A) After 4 weeks, the samples were collected for an analysis of the expression levels of ACAN and COL2 mRNA by species-specific qPCR. RNA samples were extracted from pellets ( $n=3$ per MSC donor). Relative expression levels were obtained by the normalization of human or bovine-specific signals to cross species-specific GAPDH. For human-specific genes, the values are relative amounts to the $100 / 0$ hMSC/bPC group. For bovine-specific genes, the values are relative amounts to the $0 / 100 \mathrm{hMSC} / \mathrm{bPC}$ group. Data are presented as an average of 3 BM-MSCs donors \pm SD. (B) After 4 weeks of culture, a species-specific qPCR on genomic GAPDH was performed after DNA analysis ( $n=6$ per donor). Data represent the average of 3 BM-MSC donors \pm SD. (C) After 2 days of culture, proliferation was assessed using an EdU assay. In this experiment, bPCs were labeled with CM-DiI (red). EdU incorporation into newly synthesized DNA was visualized by Alexa 488 (green), and nuclei were counterstained with Hoechst 33342 (blue) on $10 \mu \mathrm{M}$ freeze sections. Pictures show the results of a representative BM-MSC donor out of the 3 donors tested. Scale bar $=100 \mu \mathrm{m}$. (D) Quantification of EdU-positive cells. The initial ratios of hMSC and bPC are indicated by bar colors. Data from 3 donors of the BM-MSCs measured in triplicate were analyzed for statistic significance. Double asterisk represents $p<0.01$. NS, Not Significant. Error bar reflects SD. ACAN, Aggrecan; qPCR, quantitative polymerase chain reaction; GAPDH, glyceraldehyde 3-phophate dehydrogenase. Color images available online at www.liebertonline.com/tea

contents of each pellet were then determined by chemospectrophotometric and fluorescent assays (Fig. 4). MSCs from different sources performed in a similar manner in GAG formation when co-cultured with hPCs. An average of 3 donor pairs of MSCs and hPCs showed that co-culture pellets contained more GAG than 100\% chondrocyte pellets, when normalized to the initial seeding percentage of hPCs.

\section{Co-culture of hPCs and MSCs promotes chondrocyte proliferation independent of the MSCs origin}

EdU incorporation and cell tracking were used to investigate cell proliferation in the pellets. At day 2 after cell seeding, the EdU-positive cells were detected in all conditions tested (Fig. 5A). Quantitative data are shown in Figure $5 \mathrm{~B}$ and C. Percentages of EdU-positive MSCs in co-culture pellets were close to those of $100 \%$ MSCs. No significant differences were observed. Percentages of proliferating hPCs in co-culture pellets were significantly higher as compared with $100 \%$ hPCs.

After 4 weeks of co-culture, the ratios of cells derived from hPC or MSC donors were determined by short tandem repeats analysis (Fig. 5D-F). The distinguishable loci between hPC and MSC donors are illustrated, which varied between the pairs of MSCs and hPCs. On average, these loci showed a clear change in the ratio of MSCs and hPCs compared with the initial seeding density, irrespective of the source of MSCs. Three MSC donors were tested for each co-culture combination with essentially comparable results.

\section{Discussion}

Multiple mechanisms have been postulated in order to explain increased cartilage matrix formation in the co-culture 

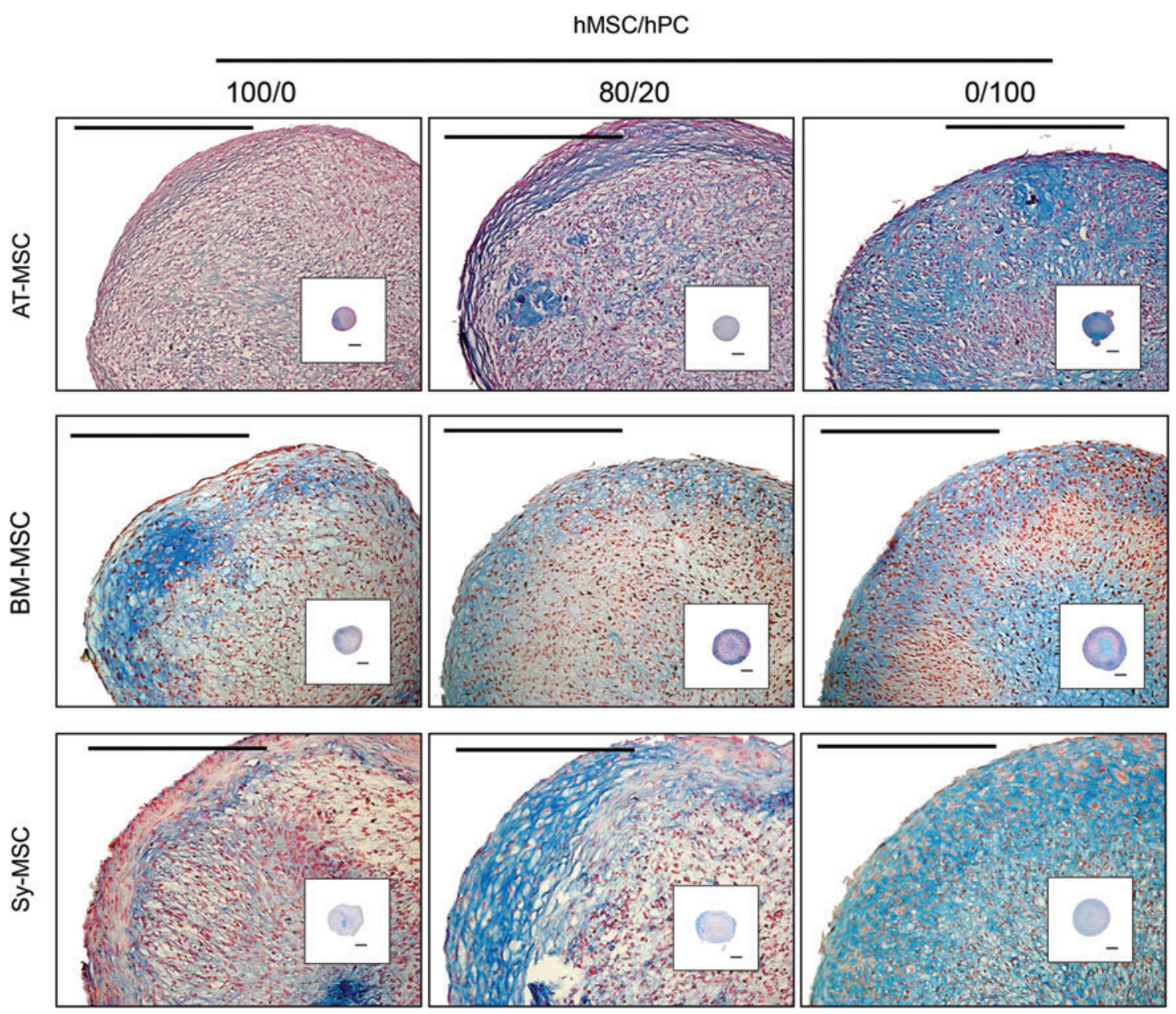

FIG. 3. GAG formation in co-culture pellets of hPCs and MSCs from different sources. Human chondrocytes were cocultured with MSCs from different sources in 3 different seeding ratios (MSC versus PC; 100:0; 80:20; 0:100) for 4 weeks in a chondrogenic differentiation medium. Alcian blue staining was performed on midsagittal paraffin sections. Nuclei were counterstained with nuclear fast red. Pictures show a representative donor of MSCs, while 3 donors were tested. Scale bar $=100 \mu \mathrm{m}$. Color images available online at www.liebertonline.com/tea

pellets of MSCs and chondrocytes. ${ }^{2}$ When this beneficial effect of the co-culture was first discovered, ${ }^{3}$ it was believed that chondrocytes stimulated the chondrogenic differentiation of MSCs, and that this contributed to the increased cartilage matrix formation in co-cultures. Several studies supported this idea. ${ }^{3,33-34}$ It was shown that a conditioned medium of chondrocytes induced the osteo-chondrogenic differentiation of MSCs, ${ }^{33,35}$ and co-culturing with chondrocytes in pellets induced chondrogenic gene expression in MSCs. ${ }^{34}$ Results from these experiments, however, were not conclusive on the role of each cell type in the co-culture, as long-term cell tracking was not performed. Earlier, we had shown that increased cartilage formation in co-culture pellets is not due to the chondrogenic differentiation of MSCs, but is predominantly caused by MSCs exerting a trophic effect on the chondrocytes stimulating cell proliferation and matrix deposition. The effect of MSCs on chondrocytes in pellet cocultures can be discerned in various effects: (1) MSCs stimulate the proliferation of chondrocytes at an early stage of the cell pellet cultures; (2) MSCs stimulate GAG formation and extracellular matrix production by the chondrocytes in the co-culture pellets; (3) As a consequence of the increased chondrocyte proliferation, the initial seeding ratio between chondrocytes and MSCs changes over time in favor of the chondrocytes; (4) This effect is further exaggerated by the preferential cell death of MSCs in the co-culture pellets. These findings were essentially confirmed in a recent publication of Acharya et al. ${ }^{6}$

Our previous study had raised a number of additional unresolved questions: First, is the trophic effect of the MSCs in co-cultures dependent on the culture conditions? Our previous pellet co-cultures were performed in a serumcontaining medium lacking chondrogenic factors that are essential for stimulating the chondrogenic differentiation of MSCs in vitro. This raises the question whether the absence of MSC differentiation and the disappearance of MSCs over time in these co-culture experiments were the result of culture conditions that are unfavorable for MSCs. To address this issue, we have repeated our co-culture experiments in a medium that is supportive of the chondrogenic differentiation of MSCs. We have performed the experiments with 3 donors who showed the capacity to differentiate into chondrocytes, albeit in a variable degree. Our results demonstrated that BM-MSCs essentially exerted a similar trophic 
FIG. 4. Trophic effects in co-cultures are independent of the origin of the MSC. Human chondrocytes were COcultured in pellets with AT-MSCs (A), BM-MSCs (B), or Sy-MSCs (C) in 3 different ratios in the chondrogenic differentiation medium. The GAG and DNA of cell pellets ( $n=6$ per donor) were quantitatively measured 4 weeks after the culture. Ratios of MSCs and bPCs are indicated by the bar colors. Scale on the left is for "Total GAG," "GAG/DNA," and "GAG/initial \% $\mathrm{PC}$," while the scale on the right is for "DNA." Asterisk represents $p<0.05$. Data are the average of 3 donors per cell source \pm SD. AT-MSCs, adipose tissue mesenchymal stem cells; SyMSCs, synovium mesenchymal stem cells. Color images available online at www.liebertonline.com/tea
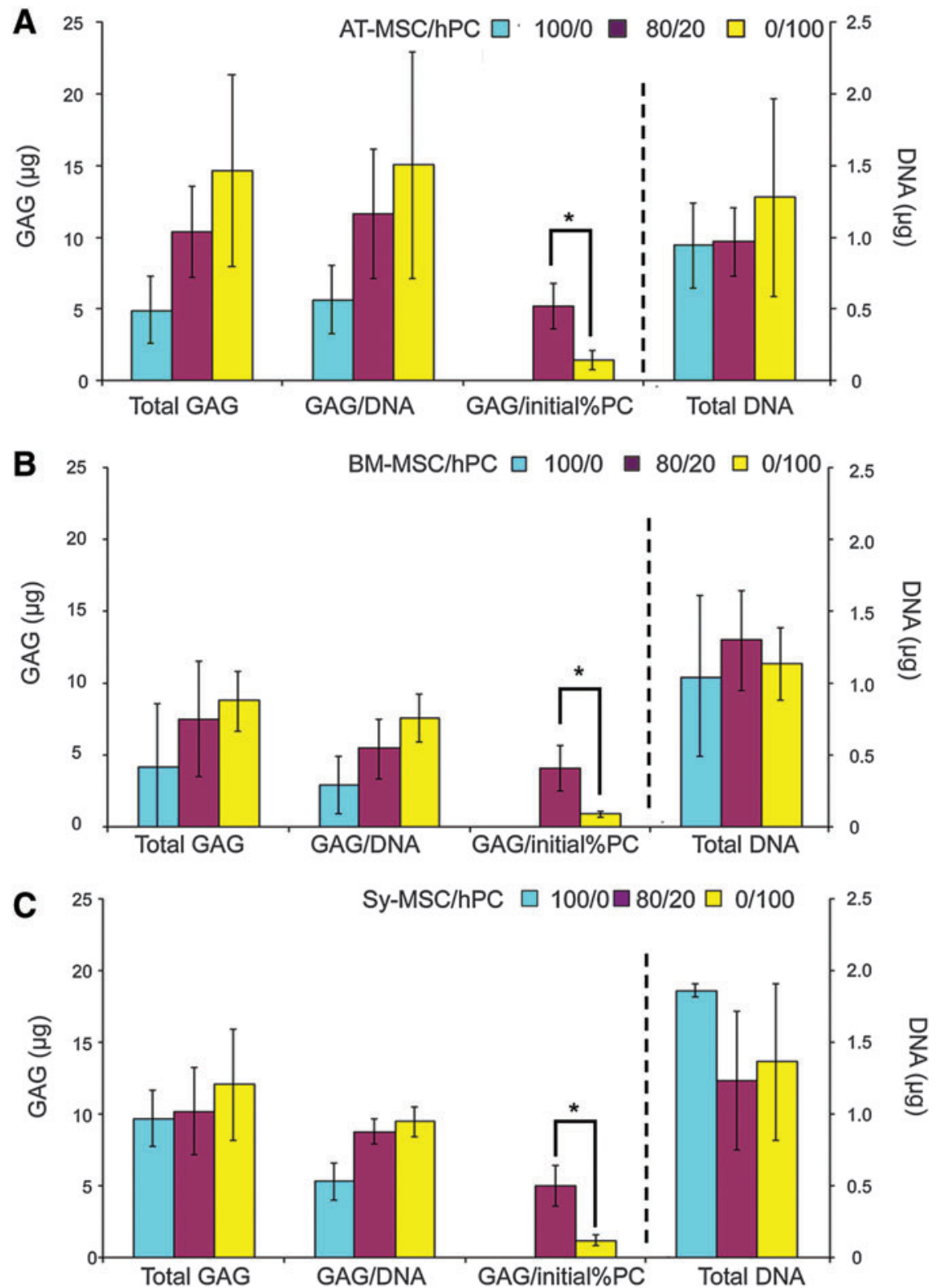

role in the co-cultures, irrespective of the culture conditions. In addition, in a chondrogenic medium, we did not observe the overt differentiation of the MSCs in chondrocytes. Based on the results of the present study, it appears that there is a common mechanism in both culture conditions, although we do find some differences in the fate of MSCs in co-cultures depending on the culture medium. The most notable difference is that the ratio change in the chondrogenic differentiation medium is not as dramatic as that in the proliferation medium. As reported, MSCs mixed with bPCs almost disappeared in the co-culture pellets in the absence of chondrogenic factors, while around $40 \%$ of MSCs remained in the co-culture pellets in the differentiation medium. ${ }^{5}$ This suggests that the MSCs cultured in pellets can survive better in a chondrogenic differentiation medium than in a proliferation medium. We did not find experimental evidence stating that the better survival of the MSCs was due to the stimulation of chondrocyte differentiation. In fact, the expression of human- specific ACAN and COL2 after 4 weeks of culture was markedly reduced in the co-culture pellets compared with the pellets of pure MSCs. In addition, the production of GAGs corrected for the initial seeding percentage of chondrocytes did not differ between the co-culture pellets cultured in a proliferation medium ${ }^{5}$ and those cultured in a chondrogenic medium (this study). Although we cannot conclude from these experiments that none of the MSCs had differentiated into chondrocytes, this effect appears to be marginal in both culture conditions. The most marked difference between both studies is that the ratio of MSCs dropped from $80 \%$ to below $5 \%$ after 4 weeks when the pellets were co-cultured in a proliferation medium, while only a reduction to $40 \%$ was seen in the chondrogenic medium. Despite this difference, similarities in both models were more common. For instance, in both models, a homogenous distribution of cartilage matrix compounds was found in the co-culture pellets even though the MSCs in the 
A
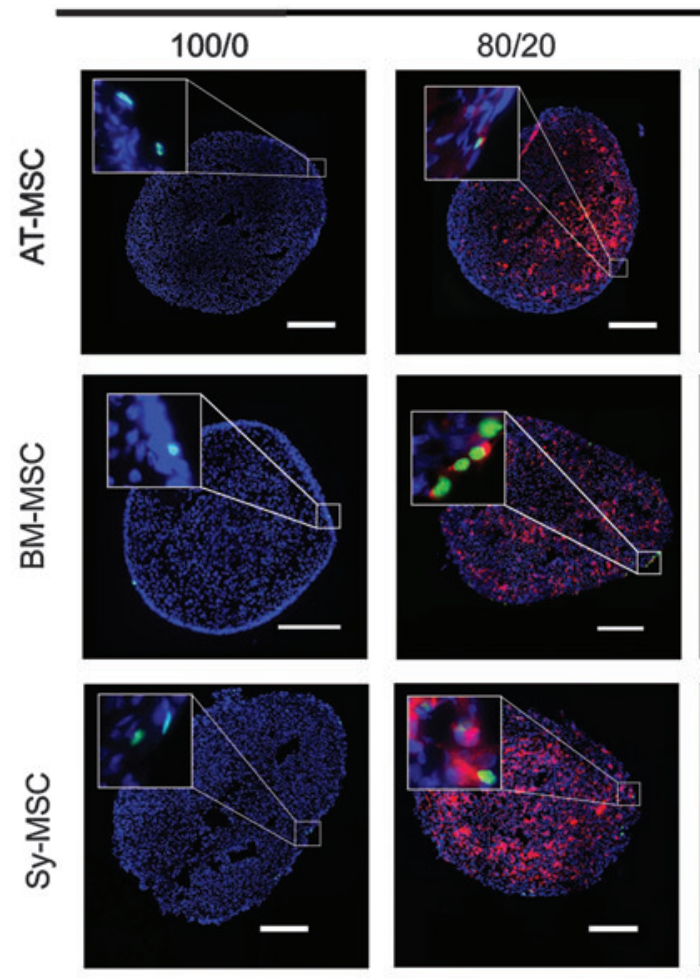

MSCs/hPCs
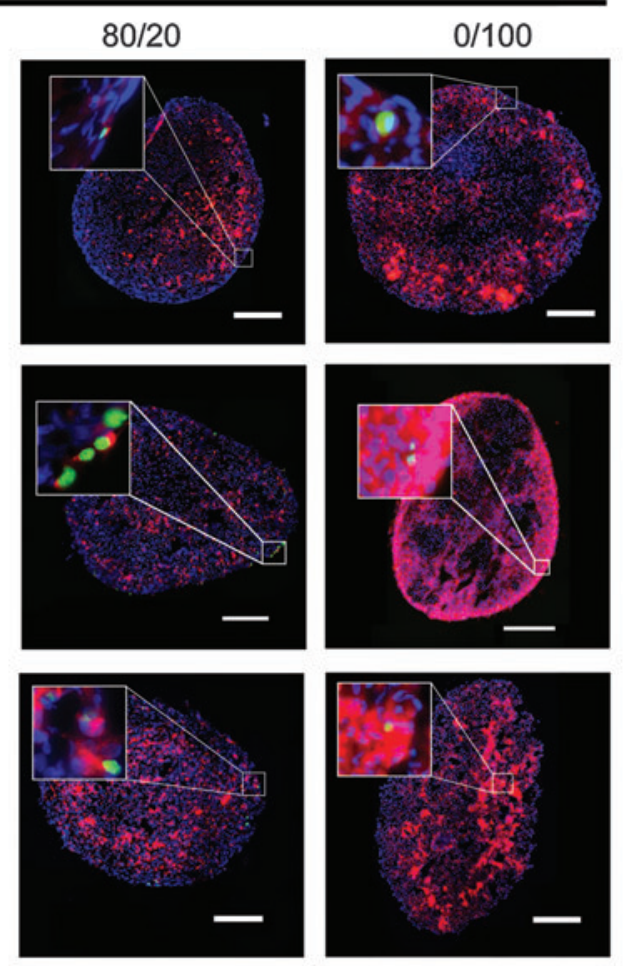

B

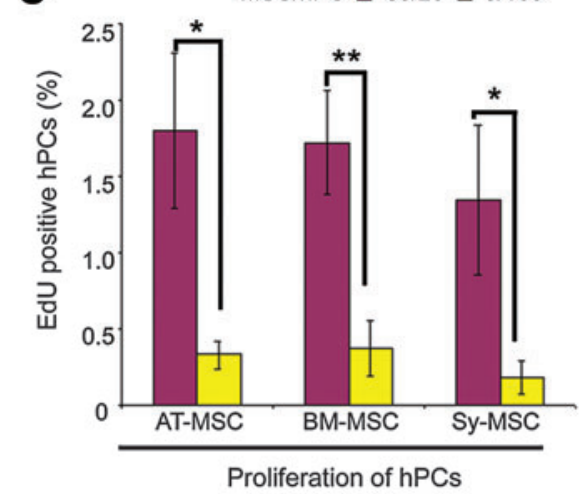

$\mathrm{MSC} / \mathrm{hPC} \square 100 / 0=80 / 20$

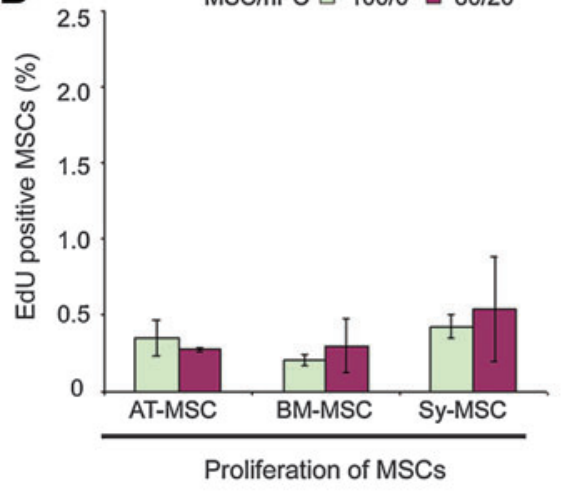

C $\mathrm{MSC} / \mathrm{hPC}=80 / 20 \square 0 / 100$
D

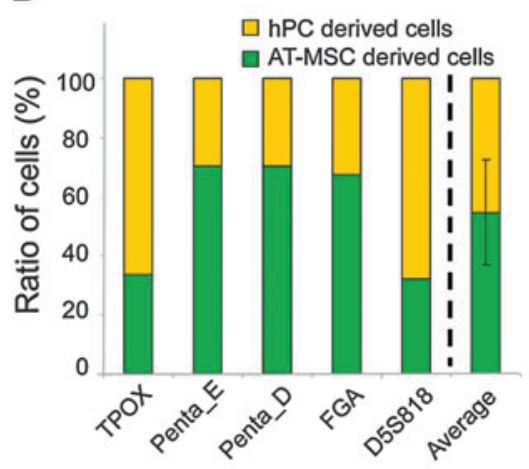

E

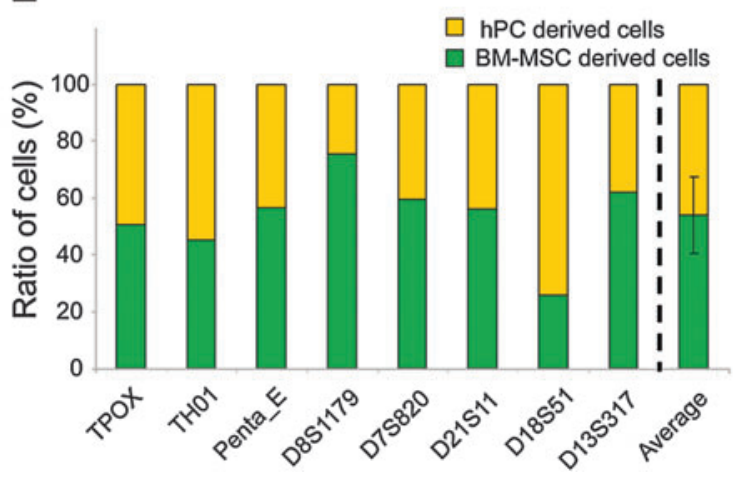

$\mathbf{F}$

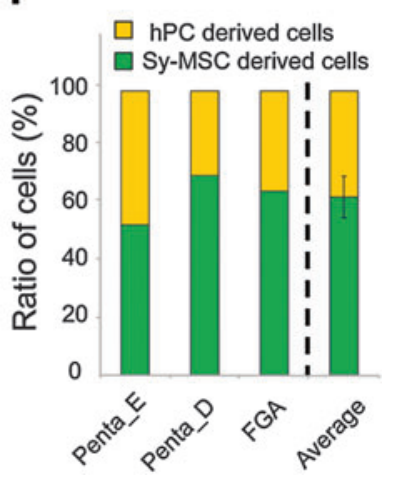

FIG. 5. MSCs from multiple sources act as trophic mediators when co-cultured with hPCs. Human chondrocytes were cocultured in pellets with AT-MSCs (A), BM-MSCs (B), or Sy-MSCs (C) in 3 different ratios in a chondrogenic differentiation medium, as described in the Materials and Methods section. (A) EdU staining was performed at day 2 to show the proliferating cells in the pellets. hPCs were labeled with CM-DiI (red). The EdU incorporation into newly synthesized DNA was visualized by Alexa 488 (green). The nuclei were counterstained with Hoechst 33342 (blue). The sources of MSCs are indicated on the left. Pictures are representative for each condition ( 3 pellets analyzed per donor) and are $10-\mu \mathrm{m}$ mid-sagittal freeze sections. The insets are magnifications of the boxed areas. Scale bar $=100 \mu \mathrm{m}$. (B) and (C) Quantification of EdU-positive MSCs (B) or hPCs (C). The initial ratios of MSC and bPC are indicated by bar colors. The sources of MSCs are indicated at the bottom. Data represent the average from 3 donors of MSCs, each measured in triplicate \pm SD. Asterisk represents $p<0.05$. Double asterisk represents $p<0.01$. (D), (E), and (F) STR analysis was performed on genomic DNA isolated from the co-culture pellets of MSCs from adipose tissue (D), bone marrow (E), or synovium (F) and hPCs at an initial seeding ratio of 80:20 after 4 weeks of the culture. Only informative loci that could discriminate between the MSCs and PCs are shown. The loci are listed at the bottom of the Figure. An average of these loci is shown by the rightmost bar \pm SD. For each combination, 3 donor pairs were tested. A representative example of each donor pair of MSCs and PCs is shown. Color images available online at www.liebertonline.com/tea

co-culture pellets with chondrocytes preferentially end up at the center of the pellet, irrespective of the culture conditions. ${ }^{5}$

The second question raised by our previous study is whether the observed role of the MSCs in the co-cultures is dependent on the source of MSCs. Our previous results were obtained with BM-MSCs. However, the beneficial effects of co-cultures for cartilage matrix formation was also found in a combination of chondrocytes and a variety of other cell types, such as adipose-tissue-derived stem cells, human embryonic stem cells, fibroblasts, and meniscus cells. ${ }^{36-39} \mathrm{It}$ 
is still unclear whether MSCs derived from adipose tissue or synovium have a comparable trophic role in the co-culture. To answer this question, we report that the MSCs isolated from adipose tissue and synovial membrane had similar trophic effects and similar behavior as BM-MSCs in the coculture with chondrocytes. We show that MSCs, irrespective of their origin, stimulate chondrocyte proliferation and increase total GAG corrected for the initial seeding percentage of chondrocytes to a comparable extent. Furthermore, we show a similar decrease in the percentage of MSCs in pellet co-cultures over time. Given these remarkably similar observations, we concluded that the MSCs from various cell sources have likely exerted similar roles.

Adipose tissue has long been considered as an alternative to bone marrow as an MSCs source, as it can be obtained relatively easily in large quantities with a relatively low donor morbidity and contains a much higher frequency of MSCs, as compared with bone marrow. ${ }^{15,30}$ Piles of documents had pointed out the potential use of AT-MSCs in a variety of tissue-engineering applications. ${ }^{40-43}$ Although less studies have been performed with synovium-derived MSCs, the synovium has recently received attention as a promising cell source for cartilage tissue engineering. ${ }^{24,31,44}$ Here, we report for the first time that MSCs isolated from bone marrow, adipose tissue, and synovium behave similarly in coculture pellets with MSCs by acting as trophic mediators stimulating chondrocyte proliferation and matrix production. Our data suggest that the trophic effects of MSCs could be a general mechanism by which the MSCs from different origins orchestrate tissue function repair.

Taken together, our results demonstrate that in co-culture pellets, MSCs stimulate cartilage formation due to a trophic effect with chondrocytes rather than differentiating into chondrocytes, irrespective of the culture conditions or their origin. This implies that the trophic effect of MSCs in cocultures is a general phenomenon that may have potential implications for the use of MSCs in cartilage repair strategies.

\section{Acknowledgments}

The authors gratefully acknowledge the financial support of the TeRM Smart Mix Program of the Netherlands Ministry of Economic Affairs and the Netherlands Ministry of Education, Culture, and Science. They are grateful to Dr. Jacqueline Plass for her technical support. They also thank Ivy Wang for the language editing of this article.

\section{Disclosure Statement}

No competing financial interests exist.

\section{References}

1. Brittberg, M., Lindahl, A., Nilsson, A., Ohlsson, C., Isaksson, O., and Peterson, L. Treatment of deep cartilage defects in the knee with autologous chondrocyte transplantation. N Engl J Med 331, 889, 1994.

2. Hendriks, J., Riesle, J., and van Blitterswijk, C.A. Co-culture in cartilage tissue engineering. J Tissue Eng Regen Med 1, 170, 2007.

3. Tsuchiya, K., Chen, G., Ushida, T., Matsuno, T., and Tateishi, $T$. The effect of coculture of chondrocytes with mesenchymal stem cells on their cartilaginous phenotype in vitro. Materials Science \& Engineering C-Biomimetic and Supramolecular Systems 24, 6, 2004.

4. Gruber, H.E., Deepe, R., Hoelscher, G.L., Ingram, J.A., Norton, H.J., Scannell, B., et al. Human adipose-derived mesenchymal stem cells: direction to a phenotype sharing similarities with the disc, gene expression profiling, and coculture with human annulus cells. Tissue Eng Part A 16, 2843,2010

5. Wu, L., Leijten, J.C., Georgi, N., Post, J.N., van Blitterswijk, C.A., and Karperien, M. Trophic effects of mesenchymal stem cells increase chondrocyte proliferation and matrix formation. Tissue Eng Part A 17, 1425, 2011.

6. Acharya, C., Adesida, A., Zajac, P., Mumme, M., Riesle, J., Martin, I., et al. Enhanced chondrocyte proliferation and mesenchymal stromal cells chondrogenesis in coculture pellets mediate improved cartilage formation. J Cell Physiol 227, 88, 2012.

7. Caplan, A.I., and Dennis, J.E. Mesenchymal stem cells as trophic mediators. J Cell Biochem 98, 1076, 2006.

8. Singer, M. Trophic functions of the neuron. VI. Other trophic systems. Neurotrophic control of limb regeneration in the newt. Ann N Y Acad Sci 228, 308, 1974.

9. Bruder, S.P., Fink, D.J., and Caplan, A.I. Mesenchymal stem cells in bone development, bone repair, and skeletal regeneration therapy. J Cell Biochem 56, 283, 1994.

10. Kassis, I., Vaknin-Dembinsky, A., and Karussis, D. Bone marrow mesenchymal stem cells: agents of immunomodulation and neuroprotection. Curr Stem Cell Res Ther 6, 63, 2011.

11. Li, Y., Chen, J., Zhang, C.L., Wang, L., Lu, D., Katakowski, M., et al. Gliosis and brain remodeling after treatment of stroke in rats with marrow stromal cells. Glia 49, 407, 2005.

12. Sassoli, C., Pini, A., Mazzanti, B., Quercioli, F., Nistri, S., Saccardi, R., et al. Mesenchymal stromal cells affect cardiomyocyte growth through juxtacrine Notch-1/Jagged1 signaling and paracrine mechanisms: clues for cardiac regeneration. J Mol Cell Cardiol 51, 399, 2011.

13. Tang, Y.L., Zhao, Q., Qin, X., Shen, L., Cheng, L., Ge, J., et al. Paracrine action enhances the effects of autologous mesenchymal stem cell transplantation on vascular regeneration in rat model of myocardial infarction. Ann Thorac Surg 80, 229, discussion 36, 2005

14. Lin, Y., Liu, L., Li, Z., Qiao, J., Wu, L., Tang, W., et al. Pluripotency potential of human adipose-derived stem cells marked with exogenous green fluorescent protein. Mol Cell Biochem 291, 1, 2006.

15. Zuk, P.A., Zhu, M., Mizuno, H., Huang, J., Futrell, J.W., Katz, A.J., et al. Multilineage cells from human adipose tissue: implications for cell-based therapies. Tissue Eng 7, 211, 2001.

16. De Bari, C., Dell'Accio, F., Tylzanowski, P., and Luyten, F.P. Multipotent mesenchymal stem cells from adult human synovial membrane. Arthritis Rheum 44, 1928, 2001.

17. Vemuri, M.C., Chase, L.G., and Rao, M.S. Mesenchymal stem cell assays and applications. Methods Mol Biol 698, 3, 2011.

18. Chen, F.H., and Tuan, R.S. Mesenchymal stem cells in arthritic diseases. Arthritis Res Ther 10, 223, 2008.

19. Motaln, H., Schichor, C., and Lah, T.T. Human mesenchymal stem cells and their use in cell-based therapies. Cancer 116, 2519, 2010.

20. Balber, A.E. Concise review: aldehyde dehydrogenase bright stem and progenitor cell populations from normal tissues: 
characteristics, activities, and emerging uses in regenerative medicine. Stem Cells 29, 570, 2011.

21. Lin, Y., Tang, W., Wu, L., Jing, W., Li, X., Wu, Y., et al. Bone regeneration by BMP-2 enhanced adipose stem cells loading on alginate gel. Histochem Cell Biol 129, 203, 2008.

22. Wu, L., Zhu, F., Wu, Y., Lin, Y., Nie, X., Jing, W., et al. Dentin sialophosphoprotein-promoted mineralization and expression of odontogenic genes in adipose-derived stromal cells. Cells Tissues Organs 187, 103, 2008.

23. Wu, L., Wu, Y., Lin, Y., Jing, W., Nie, X., Qiao, J., et al. Osteogenic differentiation of adipose derived stem cells promoted by overexpression of osterix. Mol Cell Biochem 301, 83, 2007.

24. Lee, J.I., Sato, M., Kim, H.W., and Mochida, J. Transplantatation of scaffold-free spheroids composed of synoviumderived cells and chondrocytes for the treatment of cartilage defects of the knee. Eur Cell Mater 22, 275, 2011.

25. Xue, G., He, M., Zhao, J., Chen, Y., Tian, Y., Zhao, B., et al. Intravenous umbilical cord mesenchymal stem cell infusion for the treatment of combined malnutrition nonunion of the humerus and radial nerve injury. Regen Med 6, 733, 2011.

26. Park, J., Euhus, D.M., and Scherer, P.E. Paracrine and Endocrine Effects of Adipose Tissue on Cancer Development and Progression. Endocr Rev 32, 550, 2011.

27. Bhang, S.H., Cho, S.W., La, W.G., Lee, T.J., Yang, H.S., Sun, A.Y., et al. Angiogenesis in ischemic tissue produced by spheroid grafting of human adipose-derived stromal cells. Biomaterials 32, 2734, 2011.

28. Hendriks, J., Riesle, J., and Vanblitterswijk, C.A. Effect of stratified culture compared to confluent culture in monolayer on proliferation and differentiation of human articular chondrocytes. Tissue Eng 12, 2397, 2006.

29. Wu, L., Cai, X., Dong, H., Jing, W., Huang, Y., Yang, X., et al. Serum regulates adipogenesis of mesenchymal stem cells via MEK/ERK-dependent PPARgamma expression and phosphorylation. J Cell Mol Med 14, 922, 2010.

30. Jurgens, W.J., Oedayrajsingh-Varma, M.J., Helder, M.N., Zandiehdoulabi, B., Schouten, T.E., Kuik, D.J., et al. Effect of tissue-harvesting site on yield of stem cells derived from adipose tissue: implications for cell-based therapies. Cell Tissue Res 332, 415, 2008.

31. Lee, S.Y., Nakagawa, T., and Reddi, A.H. Mesenchymal progenitor cells derived from synovium and infrapatellar fat pad as a source for superficial zone cartilage tissue engineering: analysis of superficial zone protein/lubricin expression. Tissue Eng Part A 16, 317, 2010.

32. Livak, K.J., and Schmittgen, T.D. Analysis of relative gene expression data using real-time quantitative PCR and the 2(-Delta Delta C(T)) Method. Methods 25, 402, 2001.

33. Hwang, N.S., Varghese, S., Puleo, C., Zhang, Z., and Elisseeff, J. Morphogenetic signals from chondrocytes promote chondrogenic and osteogenic differentiation of mesenchymal stem cells. J Cell Physiol 212, 281, 2007.

34. Vadala, G., Studer, R.K., Sowa, G., Spiezia, F., Iucu, C., Denaro, V., et al. Coculture of bone marrow mesenchymal stem cells and nucleus pulposus cells modulate gene ex- pression profile without cell fusion. Spine (Phila Pa 1976) 33, $870,2008$.

35. Lee, J.S., and Im, G.I. Influence of Chondrocytes on the Chondrogenic Differentiation of Adipose Stem Cells. Tissue Eng Part A 16, 3569, 2010.

36. Bigdeli, N., Karlsson, C., Strehl, R., Concaro, S., Hyllner, J., and Lindahl, A. Coculture of human embryonic stem cells and human articular chondrocytes results in significantly altered phenotype and improved chondrogenic differentiation. Stem Cells 27, 1812, 2009.

37. Gunja, N.J., and Athanasiou, K.A. Effects of co-cultures of meniscus cells and articular chondrocytes on PLLA scaffolds. Biotechnol Bioeng 103, 808, 2009.

38. Hildner, F., Concaro, S., Peterbauer, A., Wolbank, S., Danzer, M., Lindahl, A., et al. Human adipose-derived stem cells contribute to chondrogenesis in coculture with human articular chondrocytes. Tissue Eng Part A 15, 3961, 2009.

39. Hendriks, J.A.A., Miclea, R.L., Schotel, R., de Bruijn, E., Moroni, L., Karperien, M., et al. Primary chondrocytes enhance cartilage tissue formation upon co-culture with a range of cell types. Soft Matter 6, 5080, 2010.

40. Witkowska-Zimny, M., and Walenko, K. Stem cells from adipose tissue. Cell Mol Biol Lett 16, 236, 2011.

41. Hildner, F., Albrecht, C., Gabriel, C., Redl, H., and van Griensven, M. State of the art and future perspectives of articular cartilage regeneration: a focus on adipose-derived stem cells and platelet-derived products. J Tissue Eng Regen Med 5, e36, 2011.

42. Wilson, A., Butler, P.E., and Seifalian, A.M. Adipose-derived stem cells for clinical applications: a review. Cell Prolif 44, 86, 2011.

43. Cherubino, M., Rubin, J.P., Miljkovic, N., Kelmendi-Doko, A., and Marra, K.G. Adipose-derived stem cells for wound healing applications. Ann Plast Surg 66, 210, 2011.

44. Mochizuki, T., Muneta, T., Sakaguchi, Y., Nimura, A., Yokoyama, A., Koga, H., et al. Higher chondrogenic potential of fibrous synovium- and adipose synovium-derived cells compared with subcutaneous fat-derived cells: distinguishing properties of mesenchymal stem cells in humans. Arthritis Rheum 54, 843, 2006.

Address correspondence to: Marcel Karperien, Ph.D.

Department of Developmental BioEngineering MIRA Institute for Biomedical Technology and Technical Medicine University of Twente P.O.box 217, 7500AE

Enschede

The Netherlands

E-mail: h.b.j.karperien@tnw.utwente.nl

Received: December 17, 2011

Accepted: March 14, 2012

Online Publication Date: April 25, 2012 